\title{
PROCEEDING
}

\section{E2F1-deficient NOD/SCID mice are an experimental model for dry mouth}

\author{
Keitaro Satoh ${ }^{1,2}$, Takanori Narita ${ }^{2}$, Hikaru Matsui-Inohara ${ }^{3}$, Tatsuro Ito ${ }^{3}$, \\ Hidenobu Senpuku ${ }^{3}$, and Hiroshi Sugiya ${ }^{2}$ \\ ${ }^{1}$ Department of Regulatory Physiology, Dokkyo Medical University School of Medicine, Tochigi, \\ Japan ; Department of Physiology, Nihon University School of Dentistry at Matsudo, Matsudo, Japan ; \\ and ${ }^{3}$ Department of Bacteriology, National Institute of Infectious Diseases, Tokyo, Japan
}

\begin{abstract}
Saliva contains a wide variety of secretory proteins, including $\alpha$-amylase, lysozyme, peroxidase, immunoglobulins, and mucins. Hyposecretion of saliva and consequent dry mouth will lead to severe dental caries, periodontal disease, and mucosal infections, resulting in degrade of quality of life. Polyposia development is one of sign usually seen in dry mouth patients. However, little is reported in dry mouth-model animal regarding the entire process of polyposia development. We investigated the behavior of polyposia in E2F1-deficient non-obese diabetic/severe combined immunodeficiency disease (NOD/SCID) mice, as a dry mouth-model. E2F1-deficient NOD/SCID mice secreted small amount of saliva under the stimulation with a cholinergic agonist, pilocarpine, compared with control mice. The frequency of water intake by E2F-1-deficient NOD/SCID mice was more than that by control mice. These results suggest that E2F-1-deficient NOD/SCID mice show a behavior similar to polyposia and are very useful experimental model of dry mouth patients. J. Med. Invest. 56 Suppl. : 260-261, December, 2009
\end{abstract}

Keywords : dry mouth, saliva, pilocarpine, polyposia, Sjögren's syndrome

Saliva is essential for the preservation of oral health. Saliva has many physiological functions, such as food digestion, antimicrobial actions, remineralization of teeth, buffering of $\mathrm{pH}$. Salivary dysfunction results in impaired food and beverage intake, host defense, and communication, all of which ultimately have an adverse influence on a person's quality of life. Sjögren's syndrome (SS), the use of drugs with anticholinergic effects and radiotherapy for head and neck cancers are the most common causes of dry mouth. However, pathophysiological feature of dry mouth has been unclear yet. We believe, therefore,

Received for publication October 15, 2009 ; accepted October 22, 2009.

Address correspondence and reprint requests to Keitaro Satoh, Department of Regulatory Physiology, Dokkyo Medical University School of Medicine, 880 kita-kobayashi, Mibu, Tochigi 3210293, Japan and Fax : +81-282-86-7835. that establishment of dry mouth-model animals is important.

NOD mice and E2F-1-deficient mice have been used as a dry mouth-model (1). E2F-1-deficient NOD mice develop SS more progressingly than NOD mice do (1). However, such dry mouth-model mice are associated with underlying disease such as diabetes. Recently, Matsui-Inohara, et al. (2) have established E2F-1-deficient non-obese diabetic/ severe combined immunodeficiency disease (NOD/ SCID) mice. In E2F-1-deficient NOD/SCID mice, the number of ducts in the salivary gland was increased compared with control mice, suggesting destruction of acinar cells. Normally, simultaneous stimulation with isoproterenol and pilocarpine provokes small volume of salivary secretion in mice. Moreover, these mice are not associated with underlying disease except SCID. Therefore, 
E2F-1-deficient NOD/SCID mice have been considered useful for study of treatment of dry mouth.

Polyposia is a clinical sign of dry mouth in human. Treatment of polyposia is a main care for dry mouth patients. Analyzing polyposia in experimental animal has been difficult because of lack of such animal models. However, Nakamura, et al. (3) reported that muscarinic acetylcholine receptor-knockout mice showed behavior similar to polyposia when analyzed by a novel method. Under non-invasive condition, these authors measured the frequency of water intake preceded by eating using video movie, indicating that this method is useful. We, therefore, investigated the behavior of polyposia in E2F-1-deficient NOD/SCID mice using Nakamura's methods (3).

First, we examined effects of pilocarpine on saliva secretion in E2F-1-deficient NOD/SCID mice. The volume of saliva secreted was measured by the paper plug method. E2F-1-deficient NOD/SCID mice secreted saliva upon stimulation with pilocarpine $(0.05 \mathrm{mg} / 100 \mathrm{~g}$ body weight) in a time-dependent manner; the volume of saliva secreted was about one third of that by control mice. This result was similar to the previous report (2), suggesting that E2F-1-deficient NOD/SCID mice secret less amount of saliva with a cholinergic stimulation. Next, we analyzed the behavior of polyposia according to Nakamura's method (3). We recorded video movie of prandial water drinking in E2F-1-deficient NOD/ SCID mice which had been fasted overnight. The frequency and time of water intake in E2F-1-deficient NOD/SCID mice were almost double compared with control mice, suggesting that E2F-1-deficient NOD/SCID mice have behavior of polyposia.
In conclusion, E2F1-deficient NOD/SCID mice are very useful experimental model for the trearment study of dry mouth patients. The analysis of video movie taken during prandial water drinking in mice is extremely useful for study of pathophysiological feature of dry mouth.

\section{ACKNOWLEDGEMENTS}

We thank Prof. Yoshiteru Seo (Dokkyo Medical University) for his helpful advice and discussion.

\section{REFERENCES}

1. Salam MA, Matin K, Matsumoto N, Tsuha Y, Hanada N, Senpuku H : E2f1 mutation induces early onset of diabetes and Sjögren's syndrome in nonobese diabetic mice. J Immunol 173 : 4908-4918, 2004

2. Matsui-Inohara H, Uematsu H, Narita T, Satoh $\mathrm{K}$, Yonezawa H, Kuroda K, Ito T, Yoneda S, Kawarai T, Sugiya H, Watanabe H, Senpuku H : E2F-1-deficient NOD/SCID mice developed showing decreased saliva production. Exp Biol Med 234 : 1519-1524, 2009

3. Nakamura T, Matsui M, Uchida K, Futatsugi A, Kusakawa S, Matsumoto N, Nakamura K, Manabe T, Taketo MM, Mikoshiba K : $\mathrm{M}_{3}$ muscarinic acetylcholine receptor plays a critical role in parasympathetic control of salivation in mice. J Physiol 558 : 561-575, 2004 